\title{
Development of a DLC-Coated Tool with Thin Film Thermocouples
}

\author{
Kazuaki KAMIO ${ }^{1}$, Toshiyuki OBIKAWA ${ }^{2}$, Ali BASTI ${ }^{3}$, Jun SHINOZUKA ${ }^{4}$ and Yasuhiro KAMATA ${ }^{5}$ \\ ${ }^{1}$ Department of Mechanical and Control Engineering, Tokyo Institute of Technology, Japan, kamio.k.aa@m.titech.ac.jp \\ ${ }^{2}$ Institute of Industrial Science, The University of Tokyo, Japan, obikawa@iis.u-tokyo.ac.jp \\ ${ }^{3}$ Department of Mechanical and Control Engineering, Tokyo Institute of Technology, Japan \\ ${ }^{4}$ Department of Mechanical Engineering, Ibaraki University, Japan, jshinozu@mx.ibaraki.ac.jp \\ ${ }^{5}$ Institute of Industrial Science, The University of Tokyo, Japan, kamata@iis.u-tokyo.ac.jp
}

\begin{abstract}
:
This paper describes a tool with thin film thermocouples (TFTs) developed for monitoring cutting temperature in machining of aluminum alloy. Diamond like carbon (DLC) was chosen as a protective coating material of the tool. TFTs were fabricated on the rake face of a sintered alumina tool insert using sputtering and photolithography technique. Calibration of the TFTs provided an empirical formulation of Seebeck coefficient of the TFTs. Cutting temperature was measured with TFTs at high cutting speeds. It is shown that the DLC-coated tool with TFTs can be used to identify the highest cutting speed permissible in high speed cutting.
\end{abstract}

Keywords: Cutting temperature, Thin film thermocouple, DLC-coated tool, High speed cutting

\section{Introduction}

Temperature is known to have critical influences on material processing, especially on machining. Regardless of wear morphologies, wear of cutting tool is greatly affected by temperature in the tool-chip contact area. Unusual temperature elevation causes thermal cracks, which may lead to tool chipping. Machining accuracy would be degraded due to the thermal deformations of the workpiece, cutting tool and/or machine tool. Thus many experimental methods have been proposed for measuring temperature in the tool-chip contact area: for example, tool-chip thermocouple method [1], embedded thermocouple method [2, 3] and thin pure platinum film sensor technique [4]. However, no practical temperature measurement method has been established.

Sensors in a form of thin film thermocouples (TFTs) have been developed by the authors for measuring temperature in the tool-chip contact area [5]. In this paper, tools with TFTs were developed for monitoring cutting temperature in machining of aluminum alloy. Diamond like carbon (DLC) was chosen as a material of protective coating of the tool for its quite low friction characteristic against pure aluminum and aluminum alloys. TFTs, which were composed of thin films of nichrome and nickel, were fabricated on the rake face of a sintered alumina tool insert using sputtering and photolithography techniques. Then, a hafnium oxide film was deposited on the circuits of TFTs to insulate them electrically, and finally the tool was covered with a DLC coating film. After calibration of thermoelectromotive forces of the TFTs, cutting thermometry experiments of the aluminum alloy were conducted and cutting temperatures were measured with TFTs at high cutting speeds.

\section{Working principle}

Schematic views of a tool with a TFT, application of a tool with TFTs to orthogonal cutting, and the longitudinal cross section of a tool with TFTs are shown in Figs. 1, 2 and 3, respectively. The hot junctions of thermocouples are fabricated very close to the cutting edge so that they would be just beneath the tool-chip contact area, while the cold junctions, which are terminals to the recorder, are placed away from the edge but on the tool rake face. The thermoelectromotive force of a TFT is attributed to the temperature difference between the hot junction and terminal of the TFT, $\Delta T_{\text {TFT }}$. Thus, the temperature of the hot junction $T$ is given by

$$
T=\Delta T_{\mathrm{TFT}}+T_{\text {terminal }}
$$

where $T_{\text {terminal }}$ is the terminal temperature measured with a chromel-alumel thermocouple.

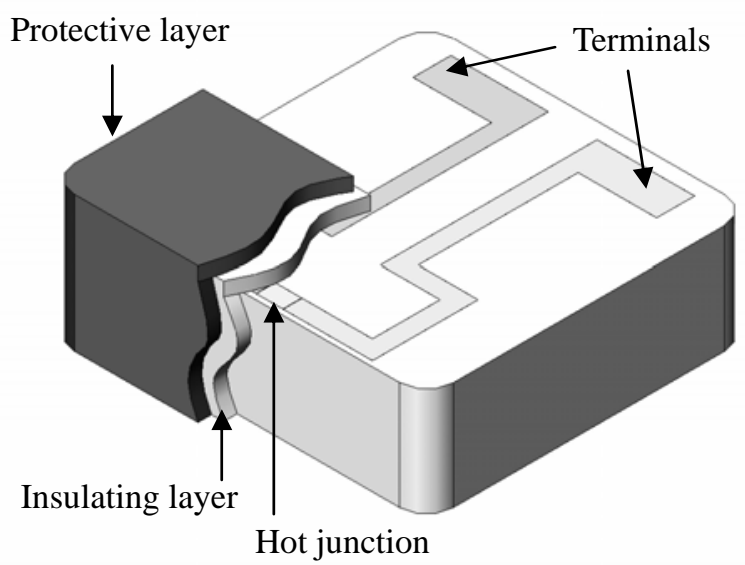

Figure 1: Schematic view of a tool with a TFT 


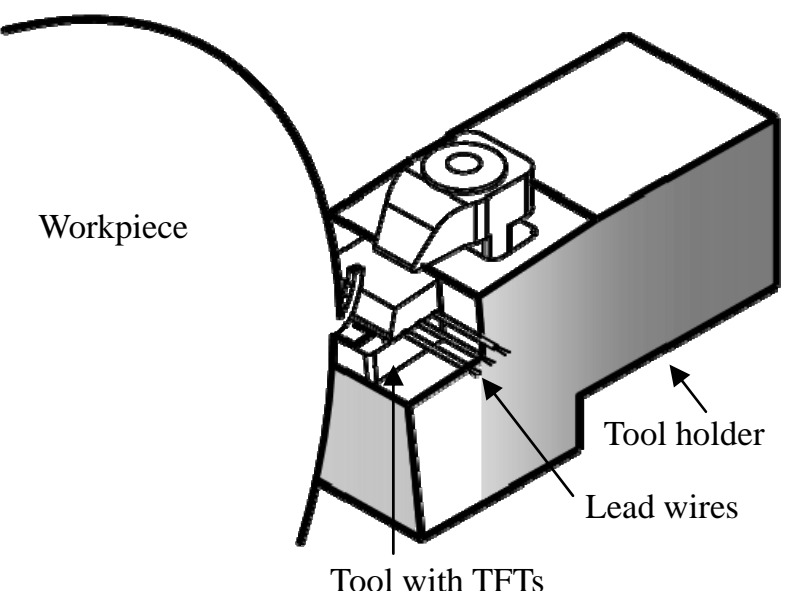

Figure 2: View of TFTs application

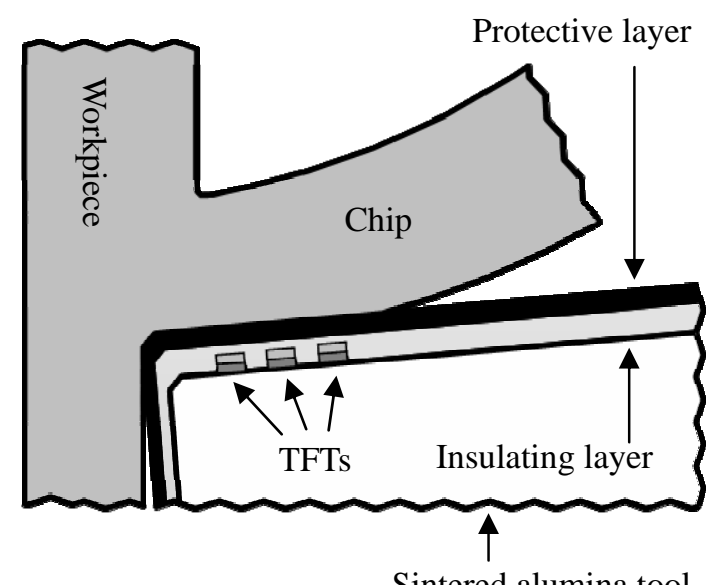

Figure 3: Schematic of longitudinal cross section of a tool with TFTs

\section{Fabrication of DLC coated tool}

The tool-chip contact area is small and subject to high pressure and high temperature during machining. Hence, the tool with TFTs must fulfill the following requirements: (1) a protective layer of the tool exhibits good wear resistance and durability; (2) the hot junctions of the TFTs are small enough and close enough to the cutting edge to measure temperatures in the contact area; and (3) the composition of the TFTs do not change during machining. To meet the above requirements, TFTs were fabricated on the rake face of a sintered alumina tool insert by sputtering and chemical etching. Then, hafnium oxide was coated to form an insulating layer, and finally a protective layer of DLC was coated.

A schematic of tool fabrication process is presented in Fig. 4. The substrate of TFTs was a sintered alumina tool insert, which had been lapped and polished to surface roughness Ra less than $0.2 \mu \mathrm{m}$, and cleaned ultrasonically with an alkaline detergent. After these preparations, a nichrome layer of a thickness of $0.5 \mu \mathrm{m}$ was deposited using DC magnetron sputtering on the left half side of the tool rake face and the overlap area near the cutting edge, where the hot junctions were to be made. This process was repeated for coating a nickel layer $0.5 \mu \mathrm{m}$ thick on the right half side of the tool rake face and the overlap area. Then, photoresist spin-coated over the whole top surface uniformly was pre-baked and exposed to ultraviolet rays through a chromium shadow mask in the shape of designed TFTs. After the development and post-baking of the photoresist with the shape of TFTs, the unnecessary parts of the $\mathrm{Ni}$ and $\mathrm{Ni}-20 \% \mathrm{Cr}$ films were removed by chemical etching. Hafnium oxide was further deposited by RF magnetron sputtering to form an insulating layer $1.8 \mu \mathrm{m}$ thick while leaving thermocouple terminals uncoated. Finally, a protective layer of DLC was coated to a thickness of $1 \mu \mathrm{m}$.

Figure 5 shows a fabricated tool before and after DLC coating. Thermocouples under the hafnium oxide layer can be seen through because transparent columnar crystals of hafnium oxide were grown up. From the
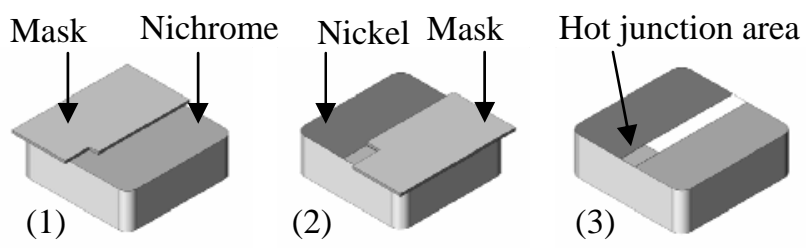

Photoresist film
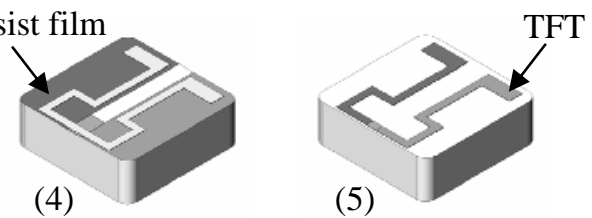

Hafnium oxide
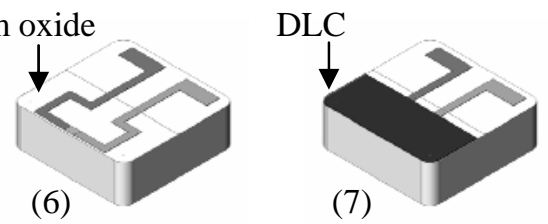

Figure 4: Process of fabricating a tool with TFT

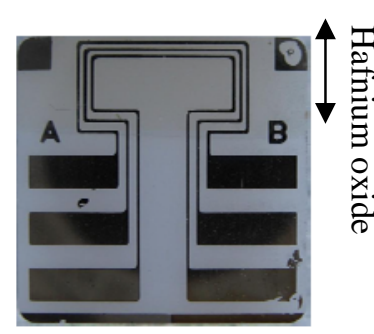

(a)

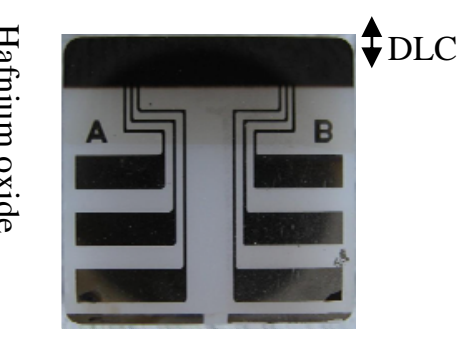

(b)
Figure 5: Fabricated tool. (a) before coating DLC and (b) after coating DLC 
cutting edge, three TFTs were named TFT1, TFT2 and TFT3. The width of hot junction is generally set at 150 $\mu \mathrm{m}$. However, the widths of hot junctions are not always fixed at the above values.

\section{Calibration of TFTs}

Seebeck coefficients of all the fabricated TFTs were obtained by calibrating all the TFTs on all the tool inserts. A setup for calibration is shown in Fig. 6. A thin steel plate, which was pressed against the rake face, was heated with a gas burner so that the plate would conduct heat to the hot junctions of the TFTs. The tool was covered with a heat insulator not to expose its surfaces to the heat radiation from the burner flame nor the cold air directly. It should be noted that the heat insulator is removed from the setup shown in Fig. 6. The temperature of the hot junction of a TFT was measured with a chromel-alumel thermocouple which was placed on the top of the steel plate right above the TFT.

As a result of calibration, it was found that Seebeck coefficients of TFTs were not constant and smaller than those of nichrome-nickel wire thermocouples. The main reasons of low Seebeck coefficients of TFTs are as follows; oxidation of the surface of a TFT and defects in the TFT have a great influence on its quantum and electrical properties; thermoelectric power depends on the quantum properties such as Fermi energy, the mean free path of electrons, etc. [6]. The relation between measured Seebeck coefficients and electrical resistances of TFTs are plotted in Fig. 7. This relation can be approximated by the following equation:

$$
S t=\exp (3.242-0.0041 R)
$$

where St is the Seebeck coefficient in $\mu \mathrm{V} / \mathrm{K}$ and $R$ the total electrical resistance of a TFT circuit in $\Omega$.

\section{Cutting experiment}

The orthogonal cutting experiment was performed with a lathe. Cutting force, thrust force and cutting temperature were measured with the measuring systems

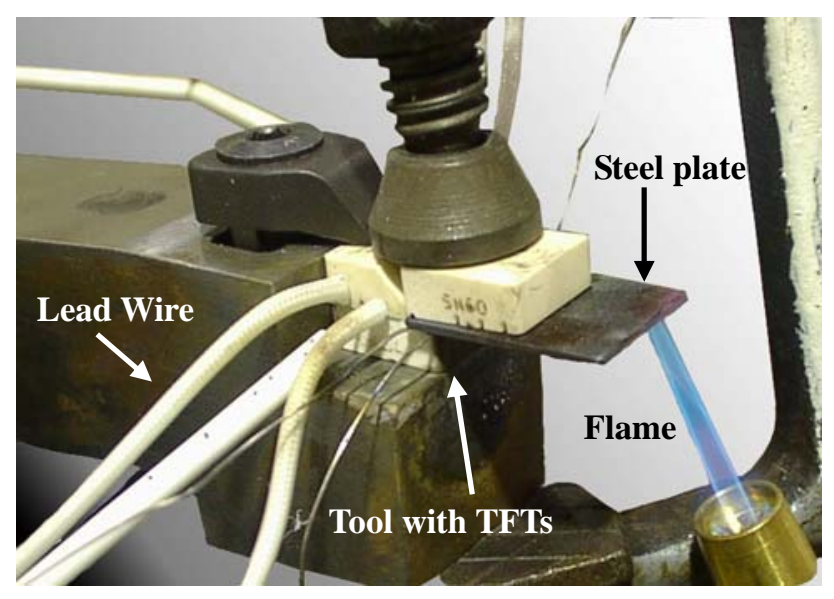

Figure 6: Experimental setup for calibration

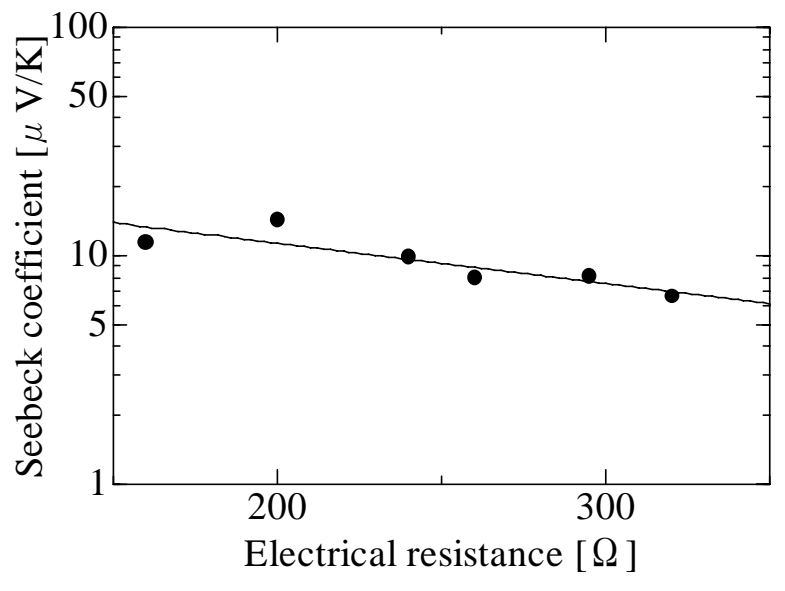

Figure 7: Experimental result of calibration

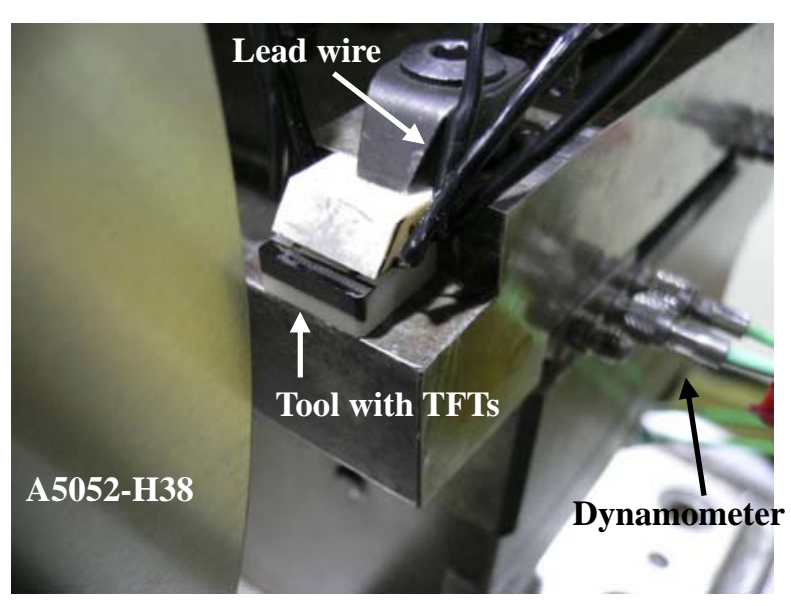

Figure 8: Experimental setup for measuring cutting temperature and forces

set up on the lathe, as shown in Fig. 8. Two piezoelectric quartz dynamometers under the base of the tool holder, charge amplifiers and a personal computer were used for the force measurement system. The thermoelectromotive forces of the TFTs and chromel-alumel thermocouples, which correspond to $T_{\mathrm{TFT}}$ and $T_{\text {terminal }}$ in Eq. (1), respectively, were transferred to the computer for the force measurement system. Then, the calibration equation (2) was used to convert the thermoelectromotive force to temperature.

The rake and relief angles of the tool are $-5^{\circ}$ and $5^{\circ}$, respectively. The workpiece was a round plate of A5052-H38 aluminum alloy $1.0 \mathrm{~mm}$ thick, which was fixed to a mandrel chucked to the spindle. Thus, cutting width was $1.0 \mathrm{~mm}$ and the feed rate was fixed at 0.10 $\mathrm{mm} / \mathrm{rev}$. Two different cutting speeds, 12 and $14 \mathrm{~m} / \mathrm{s}$ were selected to measure the cutting temperature and the cutting forces.

\section{Results and Discussion}

Data for two different cutting speeds were obtained with two different tools with DLC protective coating. Figure 9 shows cutting and thrust forces, and 
temperatures of TFT1, TFT2 and TFT3 which were placed at distances of $0.20,0.40$ and $0.60 \mathrm{~mm}$ from the cutting edge. TFT1 was in the tool-chip contact area, while TFT2 and TFT3 were outside of the contact area.

The highest temperatures measured in cutting experiment are approximately $900 \mathrm{~K}$ for cutting speed 12 $\mathrm{m} / \mathrm{s}$ and $1300 \mathrm{~K}$ for $14 \mathrm{~m} / \mathrm{s}$. Because the oxidation temperature of DLC is around $673 \mathrm{~K}$ and the melting point of aluminum is $933 \mathrm{~K}$, DLC coating cannot work effectively in high speed machining. Irregular change in temperature of TFT1 suggests that DLC coating decomposed in the course of temperature rise. Thus, the

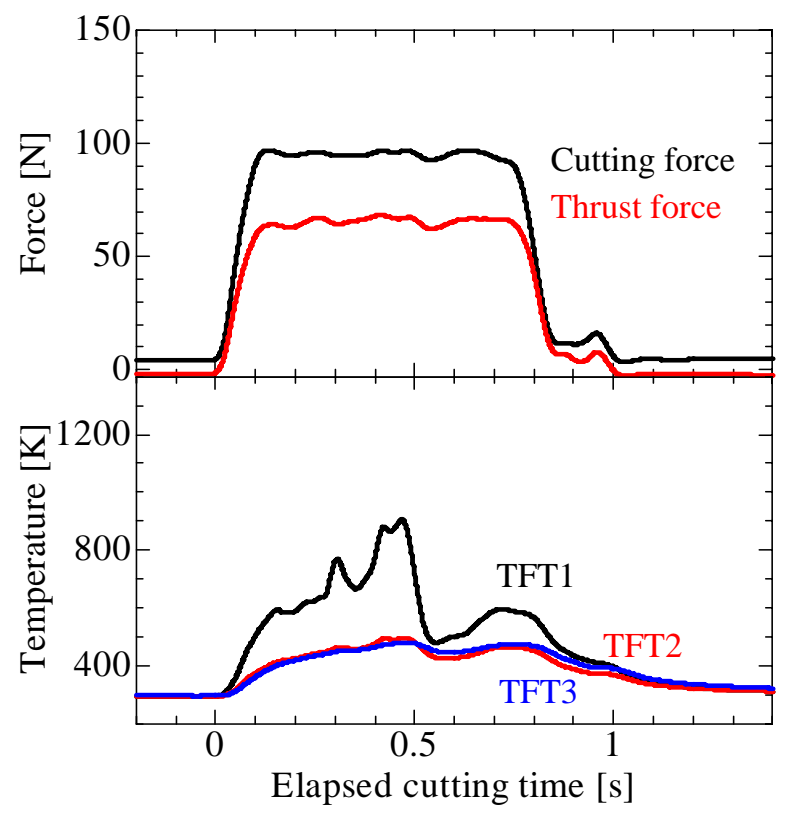

(a)

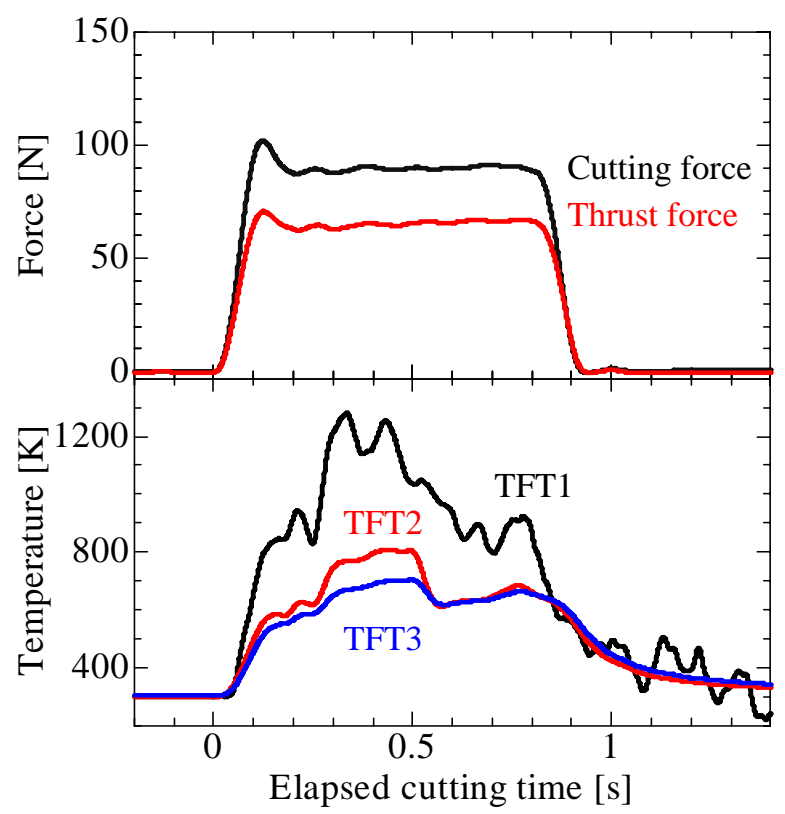

(b)

Figure 9: Experimental results for orthogonal cutting of aluminum alloy A5052-H38. (a) cutting speed $12 \mathrm{~m} / \mathrm{s}$ (b) cutting speed $14 \mathrm{~m} / \mathrm{s}$ critical cutting speed may be determined on the basis of the critical temperature of DLC coating obtained in machining aluminum alloy using a tool with TFTs.

\section{Conclusions}

In this study, TFTs fabricated on the rake face of a sintered alumina tool insert were used to measure the temperature of the tool-chip contact area in machining aluminum alloy. DC magnetron sputtering and RF magnetron sputtering were used to deposit thin films of nichrome, nickel and hafnium oxide. Photolithography and chemical etching were used to shape the thin thermocouple circuits on the tool. DLC was chosen for the protective coating material of the tool.

Calibration of the TFTs was carried out for each tool. This led to an exponential relation between the electrical resistance and Seebeck coefficient of TFTs. Orthogonal cutting thermometry experiment of A5052-H38 aluminum alloy were conducted at two different high cutting speeds. The results proved that the fabricated sensors are sensitive to the cutting temperature, and suggested that the critical temperature measured with a TFT tool would determine the highest cutting speed permissible in high speed cutting with a DLC coated tool.

\section{Acknowledgement}

The authors would like to thank Onward Ceramic Coating Co., Ltd. for coating DLC on the tools with TFTs.

\section{References}

[1] Shaw, M.C., 1984, Metal Cutting Principles, Oxford University Press, London.

[2] Usui, E., Shirakashi, T. and Kitagawa, T., 1978, Analytical Prediction of Three Dimensional Cutting Process (Part 3), Journal of Engineering for Industry, Vol. 100, pp.236-243.

[3] Obikawa, T., Matsumura, T., Shirakashi, T. and Usui, E., 1997, Wear Characteristic of Alumina Coated and Alumina Ceramic Tools, Journal of Materials Processing Technology, Vol.63, pp.211-216.

[4] Klocke, F. and Rehse, M., 1997, Intelligent Tools through Integrated Micro Systems, Production Engineering, Vol.4, pp.65-68.

[5] Basti, A., Obikawa, T. and Shinozuka, J., 2007, Tools with Built-in Thin Film Thermocouple Sensor for Monitoring Cutting Temperature, International Journal of Machine Tools and Manufacture, Vol.47, pp.793-798.

[6] Shinozuka, J., Basti, A. and Obikawa, T., Development of Cutting Tool with Built-in Thin Film Thermocouples for Measuring High Temperature Fields in Metal Cutting Processes, submitted to Journal of Manufacturing Science and Engineering. 